\title{
PEGylation of Double-Walled Carbon Nanotubes for Increasing Their Solubility in Water
}

\author{
Haiyu Nie ${ }^{1}$, Wei Guo ${ }^{1}$, Yuan Yuan ${ }^{1}$, Zengpei Dou ${ }^{1}$, Zujin Shi ${ }^{1}$, Zhen Liu ${ }^{2}$, Haifang Wang ${ }^{1,2}(\varangle)$, and \\ Yuanfang $\operatorname{Liu}^{1,2}(\bowtie)$ \\ ${ }^{1}$ Beijing National Laboratory for Molecular Sciences, College of Chemistry and Molecular Engineering, Peking University, Beijing \\ 100871, China \\ ${ }^{2}$ Institute of Nanochemistry and Nanobiology, Shanghai University, Shanghai 200444, China \\ Received: 18 September 2009 / Revised: 12 December 2009 / Accepted: 12 December 2009 \\ C The Author(s) 2010. This article is published with open access at Springerlink.com
}

\begin{abstract}
Polyethylene glycol (PEG) functionalized double-walled carbon nanotubes (DWNTs) have been synthesized by a $[2+1]$ cycloaddition reaction and characterized by transmission electron microscopy, atomic force microscopy, Raman spectroscopy, thermal gravimetric analysis, and UV-visible spectroscopy. Functionalization affords a large increase in the aqueous solubility of DWNTs. The saturated concentrations of DWNTs functionalized with diazido-terminated $\mathrm{PEG}_{800}$ (with a molecular weight of 800 ) and azido-terminated $\mathrm{PEG}_{750}$ monomethylether (with a molecular weight of 750 ) are very similar -0.36 and $0.37 \mathrm{mg} / \mathrm{mL}$ (DWNTs equivalent concentration), respectively.
\end{abstract}

\section{KEYWORDS}

Double-walled carbon nanotubes (DWNTs), functionalization, polyethylene glycol (PEG), water soluble

\section{Introduction}

Because of their novel structure and electronic properties, double-walled carbon nanotubes (DWNTs) have attracted particular attention during the past few years. Many potential applications in various fields have been explored, such as field-effect transistors, atomic force microscope (AFM) tips, filaments, energy conversion materials, and resistors [1-5].

However, like single-walled carbon nanotubes (SWNTs), their insolubility in most common solvents severely impedes the manipulation and application of DWNTs. Functionalization is a common method to solve this problem, but there have only been a few previous studies directed at DWNTs. The first example involved the covalent attachment of fluorine atoms to DWNTs [6]. Later, the reaction of DWNTs with lysine was reported [7]. However, neither of these papers mentioned the solubility of the product. Very recently, DWNTs were covalently modified with azobis-type radical initiators but the resulting product could only be well dispersed in methanol [8].

Polymers play an important role in the functionalization of carbon nanotubes, especially SWNTs, because the polymer chains may help the nanotubes dissolve efficiently in solvents and can also enhance dispersion of the nanotube bundles. One important and feasible route is sidewall functionalization via

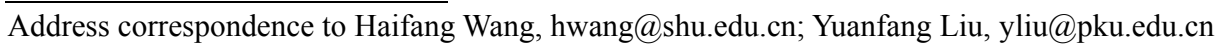


reactions with a variety of reactive molecules [9-12]. Supposing DWNTs and SWNTs have similar chemical properties, addition of polymers with azido end groups might be a good choice for modification and dispersion of DWNTs.

Herein, we present the functionalization of DWNTs with two kinds of polyethylene glycol (PEG) azides [diazido-terminated PEG and azido-terminated monomethylether] by [2+1] cycloaddition. Both PEG molecules endow DWNTs with good solubility in water.

\section{Experimental}

DWNTs were synthesized by the arc discharge method [13] and purified following the protocol in reference [13]. Briefly, after oxidation in air at $400{ }^{\circ} \mathrm{C}$ for $1 \mathrm{~h}$, DWNTs were refluxed in $15 \% \mathrm{H}_{2} \mathrm{O}_{2}$ for $2 \mathrm{~h}$, $6 \mathrm{~mol} / \mathrm{L} \mathrm{HCl}$ for $12 \mathrm{~h}$, and $2.6 \mathrm{~mol} / \mathrm{L} \mathrm{HNO}_{3}$ for $12 \mathrm{~h}$ in turn. The mixture was filtered and the sediment was annealed in air at $600{ }^{\circ} \mathrm{C}$ for $1 \mathrm{~h}$ to obtain the purified DWNTs ( $p$-DWNTs). Then, $p$-DWNTs were sonicated (96 W, Bandelin) for $6 \mathrm{~h}$ in $\mathrm{HNO}_{3} / \mathrm{H}_{2} \mathrm{SO}_{4}(v: v=1: 3)$. The acid treated DWNTs ( $t$-DWNTs) were collected on a $0.45-\mu \mathrm{m}$ polycarbonate membrane and washed with deionized water until the eluate acidity reached neutral. $t$-DWNTs were dried under vacuum at $60{ }^{\circ} \mathrm{C}$.

$\mathrm{PEG}_{800}$ azide (diazido-terminated PEG with a molecular weight of 800 ) and $\mathrm{CH}_{3} \mathrm{O}-\mathrm{PEG}_{750}$ azide (azido-terminated PEG monomethylether with a molecular weight of 750) were synthesized following Ref. [14]. $100 \mathrm{mg}$ of $t$-DWNTs was dispersed in $100 \mathrm{~mL}$ of $\mathrm{N}, \mathrm{N}$-dimethylformamide (DMF) (which was distilled under reduced pressure with $\mathrm{CaSO}_{4}$ before use) under a nitrogen atmosphere by sonication for about $2 \mathrm{~h}$. Then, the suspension was heated to $160{ }^{\circ} \mathrm{C}$. $1 \mathrm{~g}$ of $\mathrm{PEG}_{800}$ azide was first dispersed in $30 \mathrm{~mL}$ of DMF by sonication and then added dropwise to the suspension of DWNTs at $160{ }^{\circ} \mathrm{C}$. The mixture was stirred and refluxed at $160{ }^{\circ} \mathrm{C}$ for $15 \mathrm{~h}$. After the reaction, the solvent was removed by distillation and the resulting product, $\mathrm{PEG}_{800}$ functionalized DWNTs (PEG $\left.{ }_{800}-\mathrm{DWNTs}\right)$, was washed several times with ethanol to eliminate the free $\mathrm{PEG}_{800}$ residue. $\mathrm{PEG}_{800}-\mathrm{DWNTs}$ precipitated in ethanol was collected by centrifugation. The same procedure was adopted for the reaction between $\mathrm{CH}_{3} \mathrm{O}-\mathrm{PEG}_{750}$ azide and $t$-DWNTs, and the final product is denoted $\mathrm{CH}_{3} \mathrm{O}$ PEG $_{750}$-DWNTs.

PEG-DWNTs were characterized by thermal gravimetric analysis (TGA), Raman spectroscopy, transmission electron microscopy (TEM), atomic force microscopy (AFM), and UV-visible spectroscopy. TGA was performed on a TA instrument Q600 SDT. Raman spectroscopy was performed on a JY Raman microscope (HR 800, France). The morphology of DWNTs was characterized by TEM (JEOL JEM-200 CX, Japan), high-resolution TEM (HRTEM) (JEOL JEM-7401, Japan), and AFM (Shimadzu SPM-9600, Japan). UV-visible spectra were recorded on a Lambda 35 spectrometer (PE, USA). X-ray photoelectron spectroscopy (XPS) spectra were recorded on an AXIS Ultra imaging photoelectron spectrometer (Kratos, Britain).

In order to obtain the saturated concentration of PEG-DWNTs in water, a certain amount of the sample was dispersed in water by sonication for $10 \mathrm{~min}$, and then centrifuged at $3000 \mathrm{r} / \mathrm{min}$ to remove any solid residue. The resulting suspension was concentrated slowly until solid appeared. After discarding the solid, the supernatant (the saturated suspension) was collected, dried, and weighed. The saturated concentration of $\mathrm{PEG}_{800}-\mathrm{DWNT}$ and $\mathrm{CH}_{3} \mathrm{O}-\mathrm{PEG}_{750}-\mathrm{DWNT}$ at room temperature was thus obtained.

\section{Results and discussion}

Figure 1 shows TEM images of $p$-DWNTs and $t$-DWNTs. $p$-DWNTs with a length of $5-10 \mu \mathrm{m}$ existed in large bundles. We failed to modify $p$-DWNTs with the functionalized PEG molecules. The poor dispersion properties of $p$-DWNTs may account for this failure. Compared with $p$-DWNTs, $t$-DWNTs are easier to disperse in solvents, and when $p$-DWNTs were replaced by $t$-DWNTs the PEGylation reaction succeeded. The $t$-DWNTs were prepared by sonication of $p$-DWNTs in a mixed acid which causes less damage to DWNTs, and therefore better retains their intrinsic properties, than refluxing in the mixed acid. The final $\mathrm{PEG}_{800^{-}}$ DWNTs and $\mathrm{CH}_{3} \mathrm{O}-\mathrm{PEG}_{750}-\mathrm{DWNTs}$ both possessed reasonable solubilities in water, forming stable darkbrown solutions. 


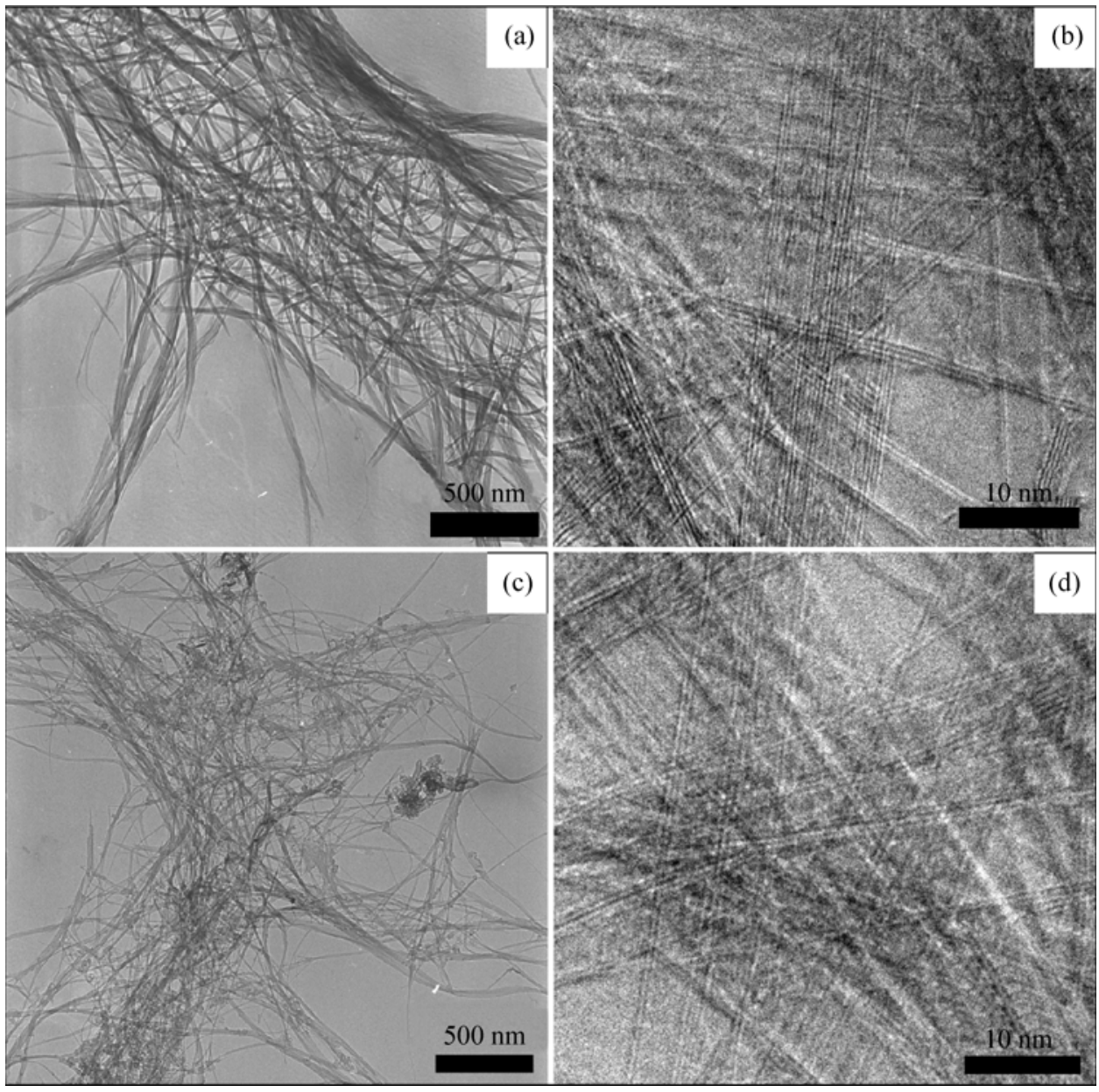

Figure 1 Representative TEM images of $p$-DWNTs and $t$ - DWNTs: (a) low-resolution TEM image of $p$-DWNTs, (b) HRTEM image of $p$-DWNTs, (c) low-resolution TEM image of $t$-DWNTs, (d) HRTEM image of $t$-DWNTs

Scheme 1 shows the reactions of $t$-DWNTs with $\mathrm{PEG}_{800}$ azide or $\mathrm{CH}_{3} \mathrm{O}-\mathrm{PEG}_{750}$ azide. We assume the reaction mechanism of the functionalization of DWNTs by the PEG azides is similar to that of the covalent functionalization of SWNTs with nitrenes [9-11]. Nitrenes contain a monovalent active nitrogen atom surrounded by a sextet of electrons. An azide can easily convert to a reactive nitrene in situ after thermally induced extrusion of $\mathrm{N}_{2}$. The nitrene may be either a singlet state (having two filled $p$-orbitals) or a triplet state (having one filled $p$-orbital and two $p$-orbitals containing unpaired electrons). Both of these can attack the nanotube sidewall to afford aziridine rings, by an electrophilic [2+1] cycloaddition and a reaction between biradicals and the $\pi$-system of the nanotube sidewalls, respectively $[9,11]$. PEG $_{800}$ azide has two azide groups, and therefore results in cross-linked

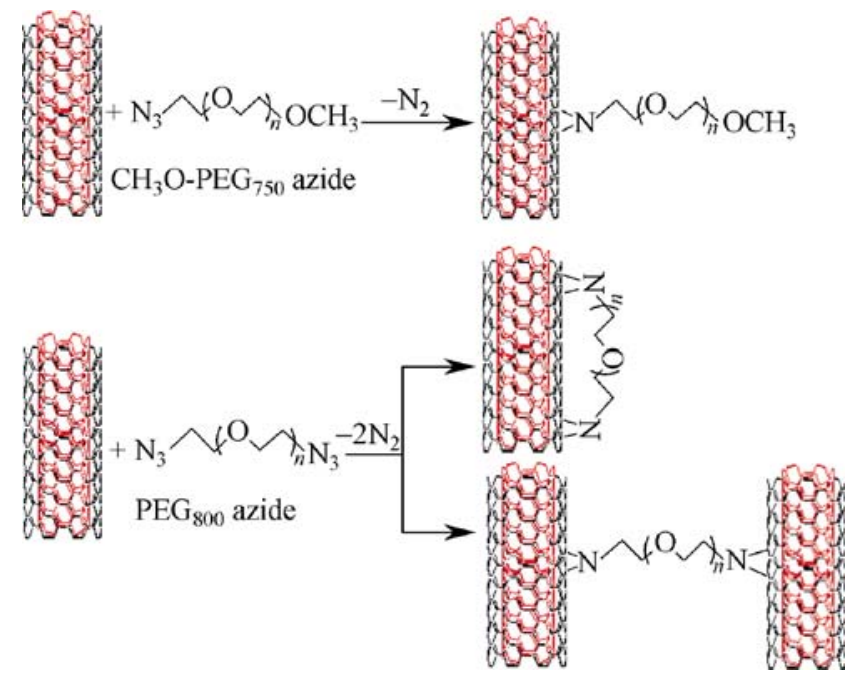

Scheme 1 The functionalization of $t$-DWNTs with PEG azides by $[2+1]$ cycloaddition 
nanotubes in $\mathrm{PEG}_{800}-\mathrm{DWNTs}$, whereas the reaction of $\mathrm{CH}_{3} \mathrm{O}-\mathrm{PEG}_{750}$ azide with DWNTs does not produce cross-linked nanotubes (Scheme 1).

$\mathrm{PEG}_{800}-\mathrm{DWNT}$ and $\mathrm{CH}_{3} \mathrm{O}-\mathrm{PEG}_{750}-\mathrm{DWNTs}$ can be seen clearly by TEM (Figs. 2(b) and 2(d)). In Fig. 2(a), the height profile of the nanotube is $2-5 \mathrm{~nm}$, corresponding to the diameter of a typical individual DWNT. This confirms the presence of individual DWNT in $\mathrm{CH}_{3} \mathrm{O}-\mathrm{PEG}_{750}-\mathrm{DWNT}$. The AFM images of $\mathrm{PEG}_{800}-\mathrm{DWNT}$ give a height of $7.0-8.5 \mathrm{~nm}$, indicating that small bundles of DWNTs are present.

Raman spectroscopy has been used to characterize the structure of DWNTs [13, 15, 16]. Figure 3 shows the Raman spectra of the four DWNT samples excited by a 532-nm laser. We observe an intense peak at $\sim 1578 \mathrm{~cm}^{-1}$ (G band), a weaker peak at $\sim 1347 \mathrm{~cm}^{-1}$ (D band), the second order $\mathrm{G}^{\prime}$ band at $\sim 2712 \mathrm{~cm}^{-1}$, and a broad peak below $200 \mathrm{~cm}^{-1}$, which are all characteristic Raman signals of DWNTs. The peak below $200 \mathrm{~cm}^{-1}$ is the radial breathing mode (RBM) of DWNTs, the frequency of which $\left(\omega_{\mathrm{r}}\right)$ is inversely proportional to the inner diameter $(d)$ of the DWNTs according to the equation $\omega_{\mathrm{r}}=6.5+223.75 / d[13,15]$. The Raman shifts and the corresponding nanotube diamaters are listed in Table S-1 in the Electronic Supplementary Material (ESM). The outer and inner diameters of $t$-DWNTs and $p$-DWNTs are in the ranges $2.02-3.56 \mathrm{~nm}$ and $1.26-2.80 \mathrm{~nm}$, respectively. The ratios of the intensity of the $G$ and $D$ bands $\left(I_{\mathrm{G}} / I_{\mathrm{D}}\right)$ for $p$-DWNTs and $t$-DWNTs are 7.3 and 3.5, respectively. Acid treatment results in an increase in the number of defects in the nanotube lattice, and therefore the $\mathrm{D}$ band intensity increases. After functionalization, the $I_{\mathrm{G}} / I_{\mathrm{D}}$ ratios of DWNTs decrease from 3.5 to 2.9 for PEG $_{800}-$ DWNTs

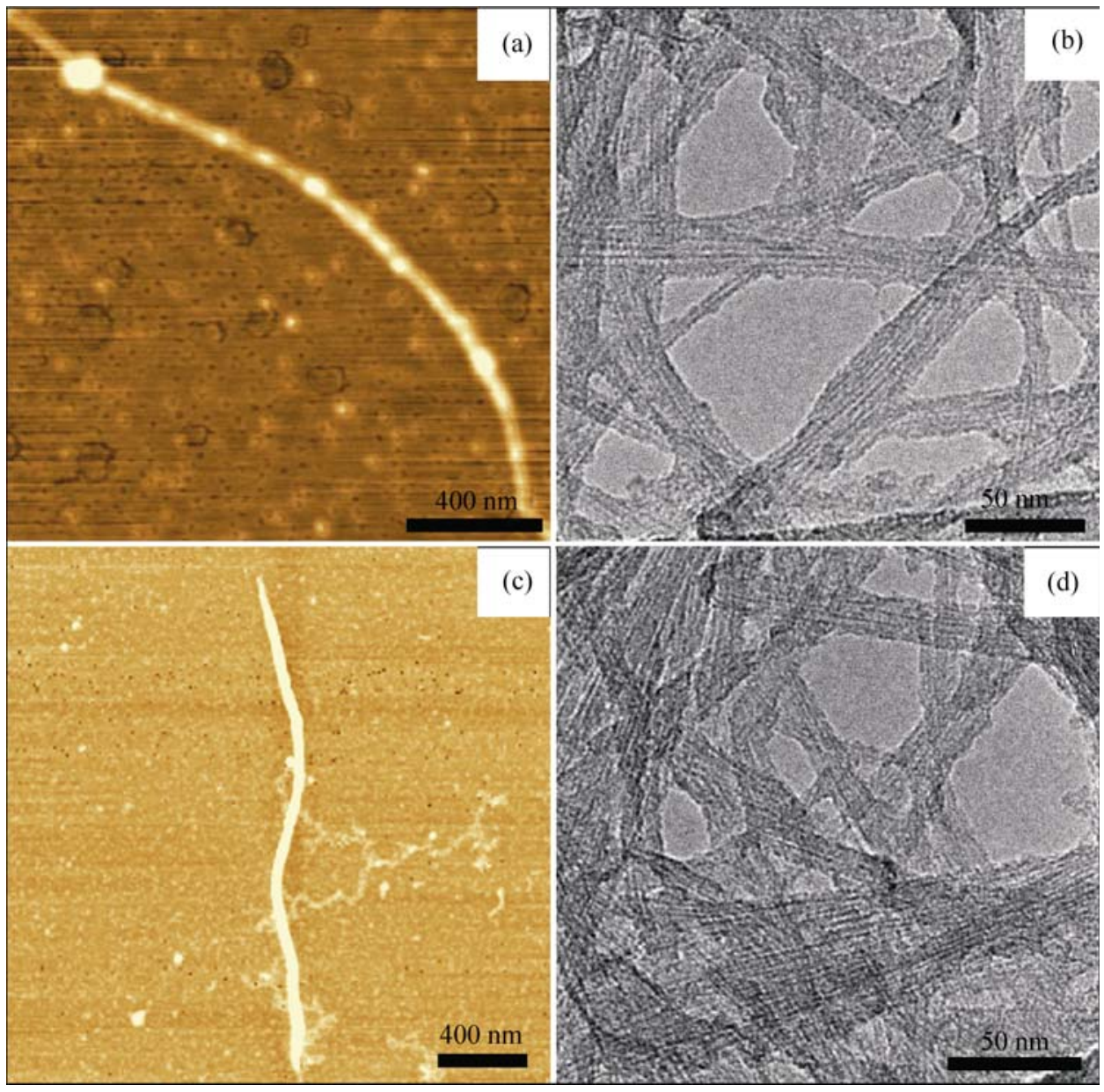

Figure 2 AFM images of (a) $\mathrm{CH}_{3} \mathrm{O}-\mathrm{PEG}_{750}-\mathrm{DWNTs}$ and (c) $\mathrm{PEG}_{800}-\mathrm{DWNTs}$; HRTEM images of (b) $\mathrm{CH}_{3} \mathrm{O}-\mathrm{PEG}_{750}-\mathrm{DWNT}$ and (d) PEG $_{800}-\mathrm{DWNTs}$ 


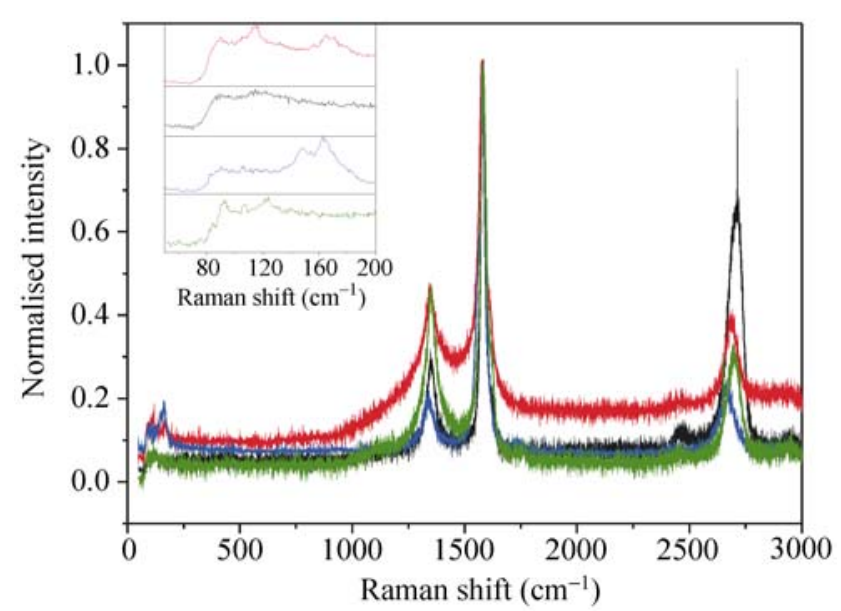

Figure 3 Raman spectra of $\mathrm{PEG}_{800}-\mathrm{DWNTs}$ (red), $\mathrm{CH}_{3} \mathrm{O}-\mathrm{PEG}_{750}$ DWNTs (black), $p$-DWNTs (blue), and $t$-DWNTs (green) excited by a $532-\mathrm{nm}$ laser. The $\mathrm{G}$ band intensity is set as $100 \%$ and the Raman spectra are normalized accordingly

and to 2.3 for $\mathrm{CH}_{3} \mathrm{O}-\mathrm{PEG}_{750}$-DWNTs, respectively. The decrease in the $I_{\mathrm{G}} / I_{\mathrm{D}}$ ratio and the increase in the $\mathrm{G}$ bandwidth after functionalization indicate a further increase in the number of defects in the nanotube lattice. This is due to the introduction of covalently bound moieties to the nanotube framework.

To evaluate the amount of polymer grafted to DWNT, TGA was performed from room temperature to $600{ }^{\circ} \mathrm{C}$ in an atmosphere of nitrogen (Fig. 4). Between 100 and $250{ }^{\circ} \mathrm{C}$, the $p$-DWNTs sample showed no weight loss, while $t$-DWNTs underwent about $5 \%$ weight loss that could be attributed to the detachment of carbonyl groups and loss of adsorbed water. TGA analysis indicated that the weight losses for $\mathrm{PEG}_{800}$ and $\mathrm{PEG}_{750}$ monomethylether occurred from 250 to $400{ }^{\circ} \mathrm{C}$ (Fig. 4). For both $\mathrm{PEG}_{800}-\mathrm{DWNTs}$ and $\mathrm{CH}_{3} \mathrm{O}-$ $\mathrm{PEG}_{750}$-DWNTs, there are two obvious processes of weight loss in their TGA curves: one occurs at temperatures less than $250{ }^{\circ} \mathrm{C}$, similar to that for $t$-DWNTs, and the other is between 250 and $400{ }^{\circ} \mathrm{C}$, which matches the temperature range for PEG decomposition. The TGA data therefore suggest that PEGylation does not occur at the defective sites of the nanotube surface.

Since the decomposition of $\mathrm{PEG}_{800}$ and $\mathrm{PEG}_{750}$ monomethylether occurs between 250 and $400{ }^{\circ} \mathrm{C}$ (Fig. 4), the weight loss difference between PEGDWNTs and $t$-DWNTs within this temperature range is a measure of the content of grafted polymer PEG.

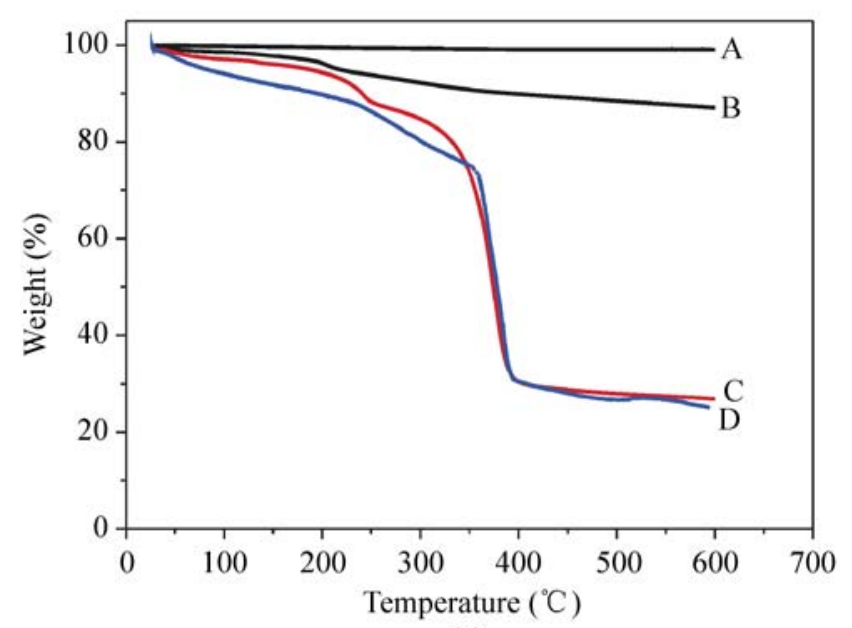

(a)

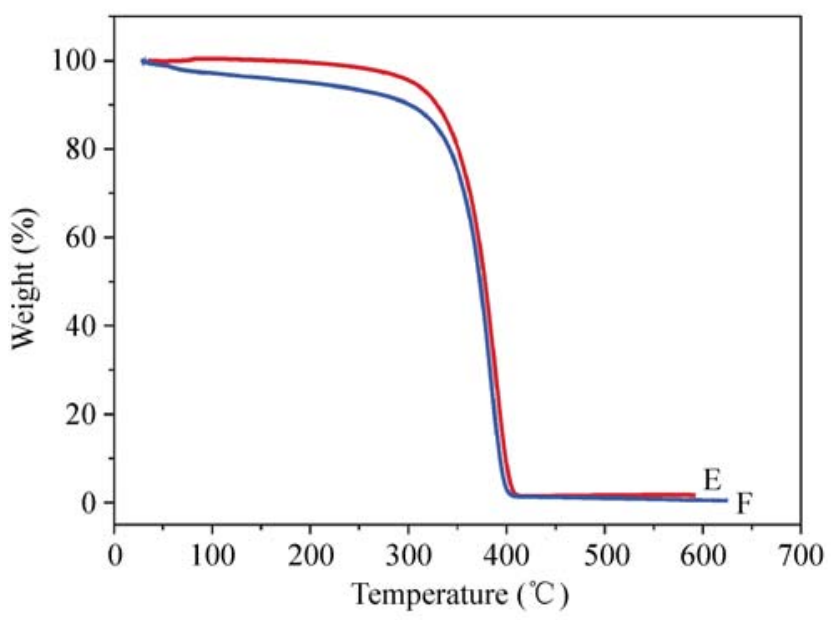

(b)

Figure 4 TGA traces for (A) $p$-DWNTs, (B) $t$-DWNTs, (C) $\mathrm{PEG}_{800}$-DWNTs, (D) $\mathrm{CH}_{3} \mathrm{O}-\mathrm{PEG}_{750}$-DWNTs, (E) $\mathrm{PEG}_{800}$, and $(\mathrm{F}) \mathrm{CH}_{3} \mathrm{O}-\mathrm{PEG}_{750}$

According to Fig. 4, the content of $\mathrm{PEG}_{800}$ in $\mathrm{PEG}_{800^{-}}$ DWNTs is about $58 \mathrm{wt} . \%$. This suggests that there is one $\mathrm{PEG}_{800}$ attached for every 48 DWNT carbon atoms in PEG $_{800}$-DWNTs (see the ESM for details of the calculation), whilst the content of $\mathrm{CH}_{3} \mathrm{O}-\mathrm{PEG}_{750}$ attached to DWNTs is about $60 \mathrm{wt} . \%$, indicating one $\mathrm{CH}_{3} \mathrm{O}-\mathrm{PEG}_{750}$ per $42 \mathrm{DWNT}$ carbon atoms.

The resulting PEG-DWNTs have fairly good solubility in water. Figure 5 shows photographs of suspensions of various DWNT samples. Samples of $\mathrm{PEG}_{800}$ and $\mathrm{PEG}_{750}$ monomethylether just simply mixed with $t$-DWNTs are shown for comparison. The five samples were sonicated in water for $10 \mathrm{~min}$. $t$-DWNTs could not be dispersed in water, therefore settled out immediately after sonication. The same 


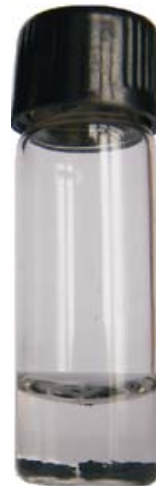

(a)

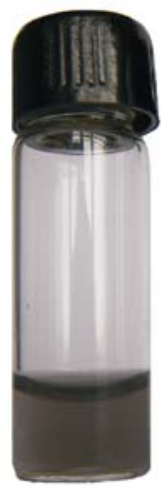

(b)

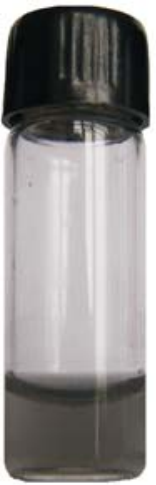

(c)

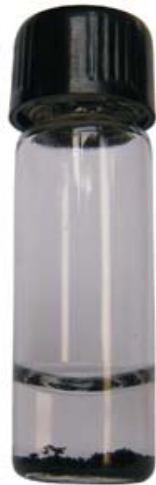

(d)

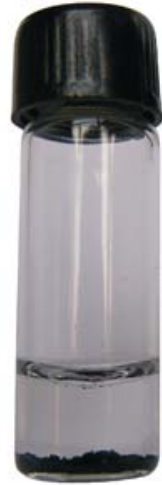

(e)
Figure 5 Photographs of various DWNTs in water: (a) $t$-DWNTs, (b) $\mathrm{CH}_{3} \mathrm{O}-\mathrm{PEG}_{750}-\mathrm{DWNTs}$, (c) $\mathrm{PEG}_{800}-\mathrm{DWNTs}$, (d) $\mathrm{CH}_{3} \mathrm{O}-$ $\mathrm{PEG}_{750} / t$-DWNTs mixture, (e) $\mathrm{PEG}_{800} / t$-DWNTs mixture. Both PEG-DWNTs suspension samples are shown after standing undisturbed for three weeks

non-dispersion phenomenon was found for the $\mathrm{CH}_{3} \mathrm{O}-\mathrm{PEG}_{750} / t-\mathrm{DWNT}$ and $\mathrm{PEG}_{800} / t$-DWNTs mixtures However, the suspensions of both PEG-DWNTs were much more stable. After centrifugation at $3000 \mathrm{r} / \mathrm{min}$ for $10 \mathrm{~min}$, both supernatants were a homogenous darkbrown color and remained stable for over three weeks without any discernible sedimentation. The saturated concentration of PEG $_{800}$-DWNTs was $0.86 \mathrm{mg} / \mathrm{mL}$ (corresponding to a DWNTs equivalent concentration of $0.36 \mathrm{mg} / \mathrm{mL}$ ). The saturated concentration of $\mathrm{CH}_{3} \mathrm{O}-$ PEG $_{750}-$ DWNTs was $0.93 \mathrm{mg} / \mathrm{mL}$, and its DWNTs equivalent concentration $(0.37 \mathrm{mg} / \mathrm{mL})$ is very close to that of $\mathrm{PEG}_{800}-\mathrm{DWNTs}$.

Figure 6 shows the UV-visible spectra of $\mathrm{PEG}_{800}-$ DWNTs in water. The intense peak of DWNTs at $265 \mathrm{~nm}$ is clearly observed. The absorbance of $\mathrm{PEG}_{800}-$ DWNTs is dependent on its concentration in a linear fashion (Fig. 6 inset), following the Beer-Lambert law. This further indicates that $\mathrm{PEG}_{800}-\mathrm{DWNTs}$ form a homogeneous water suspension. Similar UV-visible profiles of $\mathrm{CH}_{3} \mathrm{O}-\mathrm{PEG}_{750}$-DWNTs were obtained (data not shown).

In XPS analysis, the dominant $C 1$ s peak at a binding energy of ca. $285 \mathrm{eV}$ and $\mathrm{O} 1$ s peak at $533 \mathrm{eV}$ were observed in all four samples (Fig. S-1 in the ESM). For the PEG-DWNTs samples, a weak signal for $\mathrm{N} 1 \mathrm{~s}$ at $401 \mathrm{eV}$ is detected. This indicates the presence of nitrogen-carbon bonds, resulting from the PEGylation of DWNTs. The lowest O/C ratio is obtained in the $p$-DWNTs sample (Table S-2 in the

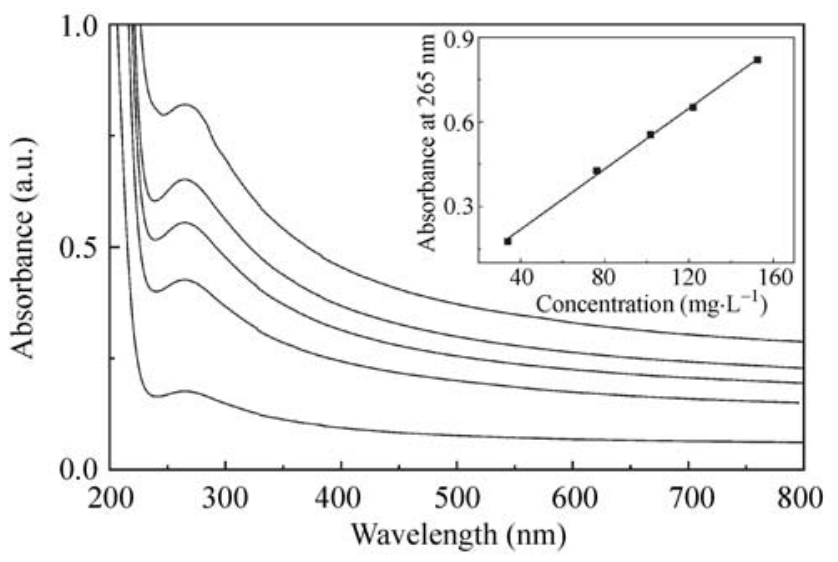

Figure 6 UV-visible absorption spectra of $\mathrm{PEG}_{800}-\mathrm{DWNT}$ at different concentrations in water. Shown in the inset is the linear dependence of absorption on suspension concentration

ESM). After acid treatment, the $\mathrm{O} / \mathrm{C}$ ratio of $t$-DWNTs increases because of the presence of O-containing groups on the surface of the nanotubes. The O/C ratios of the PEGylated DWNTs are much higher than those of $p$-DWNTs and $t$-DWNTs, because of the PEG molecules grafted to DWNTs.

Functionalization with the PEG derivatives helps the manipulation and application of DWNTs by improving the solubility of DWNTs in solvents. To date, only a few reports of DWNTs have been published, and their solubility in aqueous solution was not mentioned [6-8]. Although refluxing in the mixed acids helps to disperse DWNTs in aqueous solutions, such a process produces many defects on the surface of DWNTs, probably leading to severe damage of the nanotubes. The PEGylation method we used here occurs on the intact surface of carbon nanotubes. Therefore, it yields less damage of the DWNTs and thus retains their intrinsic properties. PEGylation also obviously endows DWNTs not only with very good solubility and stability in water, but also stability in salt solutions, such as $0.9 \% \mathrm{NaCl}$. Finally, PEG molecules have very good biocompatibility. Thus, PEGylation makes applications of DWNTs in biological fields a real possibility.

\section{Conclusions}

We have demonstrated an efficient approach to functionalize DWNTs with two kinds of PEG azides 
( PEG $_{800}-$ DWNTs and $\mathrm{CH}_{3} \mathrm{O}-\mathrm{PEG}_{750}-\mathrm{DWNTs}$ ) using $[2+1]$ cycloaddition. Both resulting PEG-DWNTs can be easily dispersed in water, forming highly stable suspensions. The resulting aqueous solubility (DWNTs equivalent concentration) of $\mathrm{PEG}_{800}$-DWNTs is $0.36 \mathrm{mg} / \mathrm{mL}$, whilst that of $\mathrm{CH}_{3} \mathrm{O}-\mathrm{PEG}_{750}-\mathrm{DWNTs}$ $0.37 \mathrm{mg} / \mathrm{mL}$. This new approach of functionalization will definitely be of benefit for the manipulation and application of DWNTs in the future.

\section{Acknowledgements}

We thank National Natural Science Foundation of China (No. 20871010), National Program on key Basic Research Project (973 Program) (Nos. 2006CB705604 and 2009CB930303), and the Shanghai Leading Academic Disciplines (No. S30109) for financial support.

Electronic Supplementary Material: Raman frequencies of the radial breathing mode $\left(\omega_{\mathrm{r}}\right)$ and the corresponding diameters $(d)$ of DWNTs, XPS spectra and analysis of composition and details of the calculation of the ratio of PEG to DWNT in PEG $_{800}-$ DWNTs are available in the online version of this article at http://dx.doi.org/10.1007/s12274-010-1014-4 and are accessible free of charge.

\section{References}

[1] Wang, S.; Liang, X. L.; Chen, Q.; Zhang, Z. Y.; Peng, L. M. Field-effect characteristics and screening in double-walled carbon nanotube field-effect transistors. J. Phys. Chem. B 2005, 109, 17361-17366.

[2] Kuwahara, S.; Akita, S.; Shirakihara, M.; Sugai, T.; Nakayama, Y.; Shinohara, H. Fabrication and characterization of high-resolution AFM tips with high-quality double-wall carbon nanotubes. Chem. Phys. Lett. 2006, 429, 581-585.

[3] Wei, J. Q.; Zhu, H. W.; Wu, D. H.; Wei, B. Q. Carbon nanotube filaments in household light bulbs. Appl. Phys. Lett. 2004, 84, 4869-4871.

[4] Wei, J. Q.; Jia, Y.; Shu, Q. K.; Gu, Z. Y.; Wang, K. L.; Zhuang, D. M.; Zhang, G.; Wang, Z. C.; Luo, J. B.; Cao, A. Y.;Wu, D. H. Double-walled carbon nanotube solar cells. Nano Lett. 2007, 7, 2317-2321.

[5] Dong, X. C.; Fu, D. L.; Xu, Y. P.; Wei, J. Q.; Shi, Y. M.;
Chen, P.; Li, L. J. Label-free electronic detection of DNA using simple double-walled carbon nanotube resistors. $J$. Phys. Chem. C 2008, 112, 9891-9895.

[6] Bulusheva, L. G.; Gevko, P. N.; Okotrub, A. V.; Lavskaya, Y. V.; Yudanov, N. F.; Yudanova, L. I.; Abrosimov, O. G.; Pazhetnov, E. M.; Boronin, A. I.; Flahaut, E. Thermal behavior of fluorinated double-walled carbon nanotubes. Chem. Mater. 2006, 18, 4967-4971.

[7] Marcolongo, G.; Ruaro, G.; Gobbo, M.; Meneghetti, M. Amino acid functionalization of double-wall carbon nanotubes studied by Raman spectroscopy. Chem. Commun. 2007, 4925-4927.

[8] Chang, J. Y.; Wu, H. Y.; Hwang, G. L.; Su, T. Y. Ultrasonication-assisted surface functionalization of doublewalled carbon nanotubes with azobis-type radical initiators. J. Mater. Chem. 2008, 18, 3972-3976.

[9] Gao, C.; He, H. K.; Zhou, L.; Zheng, X.; Zhang, Y. Scalable functional group engineering of carbon nanotubes by improved one-step nitrene chemistry. Chem. Mater. 2009, 21, 360-370.

[10] Holzinger, M.; Vostrowsky, O.; Hirsch, A.; Hennrich, F.; Kappes, M.; Weiss, R.; Jellen, F. Sidewall functionalization of carbon nanotubes. Angew. Chem. Int. Ed. 2001, 40, 4002-4005.

[11] Holzinger, M.; Steinmetz, J.; Samaille, D.; Glerup, M.; Paillet, M.; Bernier, P.; Ley, L.; Graupner, R. [2+1] cycloaddition for cross-linking SWCNTs. Carbon 2004, 42, 941-947.

[12] Bahr, J. L.; Yang, J. P.; Kosynkin, D. V.; Bronikowski, M. J.; Smalley, R. E.; Tour, J. M. Functionalization of carbon nanotubes by electrochemical reduction of aryl diazonium salts: A bucky paper electrode. J. Am. Chem. Soc. 2001, 123, 6536-6542.

[13] Qiu, H. X.; Shi, Z. J.; Guan, L. H.; You, L. P.; Gao, M.; Zhang, S. L.; Qiu, J. S.; Gu, Z. N. High-efficient synthesis of double-walled carbon nanotubes by arc discharge method using chloride as a promoter. Carbon 2006, 44, 516-521.

[14] Meunier, S. J.; Wu, Q. Q.; Wang, S. N.; Roy, R. Synthesis of hyperbranched glycodendrimers incorporating alphathiosialosides based on a gallic acid core. Can. J. Chem. 1997, 75, 1472-1482.

[15] Lyu, S. C.; Lee, T. J.; Yang, C. W.; Lee, C. J. Synthesis and characterization of high-quality double-walled carbon nanotubes by catalytic decomposition of alcohol. Chem. Commun. 2003, 1404-1405.

[16] Sugai, T.; Yoshida, H.; Shimada, T.; Okazaki, T.; Shinohara, H. A new synthesis of high-quality double-walled carbon nanotubes by high-temperature pulsed arc discharge. Nano Lett. 2003, 3, 769-773. 